\title{
FUNDAMENTAÇÃO TEÓRICO-METODOLÓGICA DA ASSISTÊNCIA AOS OSTOMIZADOS NA ÁREA DA SAÚDE DO ADULTO
}

\section{CONCEPTUAL FRAMEWORK FOR OSTOMY CARE IN THE INSTITUCIONALIZED ADULT HEALTH CARE AREA.}

Vera Lúcia Conceição de Gouveia Santos*

SANTOS, V. L. C. G. Fundamentação teórico-metodológica da assistência aos ostomizados na área da saúde do adulto.Rev.Esc.Enf.USP, v. 34, n. 1, p. 59-63, mar. 2000.

\section{RESUMO}

A autora faz uma breve revisão das bases legais, éticas e científicas que norteiam o processo de cuidar da pessoa portadora de uma ostomia, descrevendo, a seguir, algumas considerações importantes sobre esse cuidado nas diversas fases operatórias.

PALAVRAS-CHAVE: Enfermagem em Estomaterapia. Ostomia. Cuidado de Enfermagem.

\begin{abstract}
The author points out a brief revision of the legal, ethical and scientific bases of the ostomy care process and describes some important topics about caring the ostomist in the different operatory phases.
\end{abstract}

KEYWORDS: Stomal therapy nursing. Ostomy. Nursing care.

\section{INTRODUÇÃO}

A assistência a ostomizados inserida na área da saúde do adulto, tem sua fundamentação teóricometodológica no processo de cuidar em Enfermagem, estruturado, por sua vez, em princípios legais, éticos e científicos da profissão.

Embora existam diversos artigos na literatura nacional acerca do processo de cuidar de ostomizados, optou-se por realizar uma revisão inovadora ao incluir, além da abordagem técnico-científica, alguns aspectos éticos e legais que também fundamentam essa prática. Este constitue o objetivo da apresentação a seguir.

\section{BASES LEGAIS, ÉTICAS E CIENTÍFICAS DO CUIDAR DE OSTOMIZADOS}

Os fundamentos teórico-metodológicos do processo de cuidar em Enfermagem estão estruturados nos princípios legais, éticos e científicos.
A lei do Exercício Profissional de Enfermagem, de $\mathrm{n}^{\circ} 7498$ de 1986, em seu artigo 11, inciso I, que trata das competências privativas do enfermeiro, coloca em seus itens c, i, j e m, desde o planejamento, organização, coordenação, execução e avaliação da assistência de enfermagem, até a consulta de enfermagem que inclui a prescrição da assistência e as intervenções de maior complexidade técnica e científica, embasadas em conhecimentos e tomada de decisões (BRASIL, s.d.).

Do ponto de vista ético, o Código de Ética da Enfermagem (Resolução COFEN - 160) estabelece como conceitos chaves:

- o compromisso do enfermeiro como profissional, na promoção, proteção e recuperação da saúde e reabilitação, através da formação generalista ou especializada, com deveres e direitos reconhecidos; como indivíduo no exercício de sua cidadania e como ser humano, respeitando os direitos do objeto de seu cuidar enquanto ser humano, cidadão e cliente;

Enfermeira, estomaterapeuta. Prof. Dr. do Departamento de Enfermagem Médico-Cirúrgica da Escola de Enfermagem da USP. Coordenadora dos cursos de Especialização em Enfermagem e Estomaterapia. E mail: veras@usp.br. 
- as bases legais da profissão que determinam uma responsabilidade profissional direcionada para a qualidade de assistência prestada e

- envolvimento profissional e emocional com a clientela, pautado na relação de ajuda terapêutica mediada pela comunicação (CONSELHO FEDERAL DE ENFERMAGEM, s/d).

Quanto aos princípios científicos que norteiam a fundamentação teórico-metodológica do cuidar em Enfermagem, relacionam-se à implementação de método científico de resolução de problemas, que envolve, por sua vez, estratégias como a informação, instrução, motivação e treinamento do cliente. Assim como explicitadas nos aspectos legais e éticos, enfatizase a competência técnico - científica do enfermeiro como fundamental para a implementação desse sistema de cuidados.

As fases preconizadas neste método consistem em:

- avaliação das demandas assistenciais, reais e potenciais, do cliente, com estabelecimento de diagnósticos que direcionam as intervenções;

- prescrição das intervenções "cuidativas" que englobam as dimensões do saber (conhecimentos), do fazer (auto e heterocuidado) e do sentir (atitudes para a preservação ou modificação comportamental). Esta fase prevê, ainda, medidas compartilhadas ou não com demais membros da equipe interdisciplinar;

- avaliação do processo de cuidar voltado para os resultados das intervenções preconizadas e surgimento de novas demandas, retornando-se às etapas iniciais do ciclo;

- estabelecimento de critérios de alta fundamentados nos indicadores de reabilitação e qualidade de vida alcançados.

Essas considerações iniciais relativas às bases legais, éticas e científicas que fundamentam, teórica e metodologicamente, o processo de cuidar em Enfermagem, no seu sentido mais amplo, certamente constituem os princípios do cuidar em áreas menos genéricas como a Saúde do Adulto e, neste contexto, de modo mais específico, a clientela ostomizada.

\section{PROCESSO DE CUIDAR DO SER HUMANO PORTADOR DE OSTOMIA}

Os portadores de derivações externas intestinais e/ou urinárias, denominadas ostomias ou estomas, embora não constituam clientela expressiva do ponto de vista estatístico, em termos de Saúde Pública, e serem quantitativamente, cada vez menos significativos, merecem atenção especial dos profissionais, Serviços e Programas de Saúde, à medida que possuem problemática de um lado, na esfera fisica de alto custo para os poderes públicos e para o próprio cliente, relacionado com os gastos em dispositivos específicos, e de outro lado, na esfera psicossocial, ao influenciar profundamente na qualidade de vida individual e familiar. Ambos aspectos levam à necessidade de intervenções voltadas para a reabilitação.

Assim, os objetivos do cuidar de ostomizados, baseados em atenção integral e individualizada, estão dirigidos para a identificação de suas necessidades assistenciais, o estabelecimento do nivel de ajuda profissional exigido e o suficiente e adequado provimento de recursos para a reabilitação.

Para tanto, tornam-se elegiveis como princípios norteadores desse cuidar, a competência especializada dos profissionais e, especificamente, do enfermeiro; a reabilitação e a qualidade de vida como pano de fundo para a busca do autocuidado ou do cuidado compartilhado, onde paciente e família atuem como elementos fundamentais no processo de tomada de decisões; e o trabalho em equipe interdisciplinar, ou modernamente, transdisciplinar, visto que somente através desse pode-se abranger o individuo de maneira holística, na sua singularidade e universalidade (SANTOS, 1992; CASTLEDINE, 1993; STEGINGA, 1993; WRIGHT, 1993; WORLD COUNCIL OF ENTEROSTOMAL THERAPISTS-WCET, 1998).

Uma vez definidos alguns dos fundamentos teóricos do processo de cuidar do ostomizado, faz-se necessário delinear algumas diretrizes desse cuidar em Enfermagem, nas diversas fases operatórias, buscando sua sistematização, o que não representa traçar fórmulas ou rotinas assistenciais que descaracterizariam, tanto a autonomia e competência do enfermeiro, como a individualização da assistência, como. bases já mencionadas antes.

\section{Fase pré-operatória}

Esta fase deve priorizar o processo de avaliação do paciente nas esferas fisica e psicossocial, identificando o nível de autocuidado prévio e em vigência da doença.

Assim, no aspecto físico, além das condições globais normalmente levantadas nesta etapa, deve-se enfatizar a avaliação do estado nutricional, dos padrões prévios de eliminações urinária e/ou intestinal, existência e qualidade de alergias, condições da parede abdominal e deficiências fisicas que interfiram nas destrezas e habilidades do autocuidado como alterações visuais, articulares, uso de aparelhos, próteses, etc. (SANTOS, 1992; SMITH, 1992; CEZARETI et al, 1997).

Quanto às demandas psicossociais, é fundamental a identificação da dinâmica e relacionamento familiar, onde se evidenciam as relações com o outro significativo, qual seja o parceiro/ a mãe/ o filho ou outro; a sexualidade e os papéis exercidos, percebidos e 
atribuídos; além das atividades de vida diária, de trabalho e lazer (SANTOS, 1992; SMITH, 1992; CEZARETI et al, 1997).

Estar doente e estar hospitalizado têm significados de ruptura para o indivíduo. Ruptura da história de vida, de um ciclo que exige reorganização. Segundo STRAIN (1979) apud MILLER (1992), existem algumas reações psicológicas às doenças que precisam ser reconhecidas e valorizadas no processo de cuidar, por constituírem sérias ameaças ao autoconceito, autoestima e identidade do indivíduo, ao representarem uma discrepância entre a percepção do sujeito sobre si mesmo e sua realidade atual. A ameaça percebida à auto-estima e integridade corporal; o medo de perder amor e aprovação, o medo de perder controle sobre funções corporais que implicam na perda da independência; a culpa e o medo são algumas dessas reações que geram canalização de energia para o eudoente; mudanças de papéis e identidade; ansiedade frente ao futuro e uma série de outros sentimentos que se traduzem, basicamente, pela perda de controle/ poder sobre o ambiente e, o que é mais importante, sobre si mesmo.

Assim, os cuidados específicos nesta fase devem enfocar não só o preparo físico, nutricional, intestinal e de pele, incluindo aqui, particularmente, a demarcação da localização do futuro estoma como um fator essencial na recuperação e autonomia do autocuidado em relação ao manuseio do estorna, pele periestoma e dispositivo, mas também, especialmente, o preparo psicossocial (SANTOS, 1992; CEZARETI et al, 1997).

Michael Kelly, sociólogo inglês ileostomizado na década de 80 por doença inflamatória intestinal, tem escrito diversos trabalhos onde vem analisando, sob a ótica sócio-psicológica, as fases de sua recuperação. Em 1994, denominou de "zona de transição" esta fase de conflito intenso pelo qual o ostomizado passa, quando, ao decidir pela ostomia, mesmo antes de tê-la, vivencia duas identidades: o seu eu pessoal e sua nova identificação, publicamente atribuída (KELLY, 1994).

Portanto, o suporte emocional, passivel de ser compartilhado por toda a equipe e a identificação e desenvolvimento de mecanismos de enfrentamento que podem e devem ser "trabalhados" com o paciente, já nesta etapa, são cuidados importantíssimos neste momento (GOLDBERG, 1991; FREEDMAN, 1994). Muito mais, às vezes, do que as informações desmedidas que, ao invés de atenderem às demandas do paciente e aí sim, teriam um significado de aumentar o conhecimento e, conseqüentemente, o controle/poder sobre a situação - satisfazem unicamente as necessidades do enfermeiro em "cumprir" o seu papel de orientar/ informar(sem ouvir o outro).

Um outro aspecto do cuidar bastante importante, ainda na fase pré-operatória, tem sido atribuído à visita de ostomizado reabilitado, desde que treinado adequadamente para esta finalidade, por equipe de saúde em parceria com as associações de ostomizados, segundo programas préestabelecidos validados. Além desta exigência, este ostomizado deve ter doença de base e ostomia, similares ao paciente a ser visitado, que, por sua vez, evidentemente, deve concordar com a visita (SANTOS, 1992; CEZARETI et al, 1997).

A utilização pré-operatória de bolsa coletora após a demarcação, pelo indivíduo que será ostomizado (CEZARETI et al, 1997), é um item bastante controverso em literatura e que, ao levar o paciente a vivenciar antecipadamente a ostomia, por meio do dispositivo, pode aumentar o conflito da duplicidade de identidade, na "zona de transição" de que nos fala KELLY (1994), antecipando e recrudescendo a experiência do sofrimento.

\section{Fase trans-operatória}

A fase trans-operatória deve ser caracterizada pela confecção de um estorna tecnicamente adequado, onde não só participa o cirurgião, com sua competência especifica, mas, indiretamente, também o enfermeiro e o paciente, através da demarcação da localização do estorna, feita no período anterior.

Além deste, a colocação de dispositivo coletor adequado, imediatamente após a confecção do estorna, ainda na sala de cirurgia, é necessária não só para prevenir complicações da ferida operatória decorrentes da contaminação pelo vazamento de efluente, como também para permitir visualização constante do estorna e efluente, levando à detecção precoce de complicações na fase seguinte (SANTOS, 1992; CEZARETI et al 1997).

Tanto um quanto outro cuidado, dependem de uma relação harmoniosa de trabalho entre os elementos da equipe de enfermagem e destes com a equipe médica.

\section{Fase Pós-Operatória}

Esta fase pode ser, didaticamente, subdividida em imediata, mediata e tardia, à medida que, embora contínua, implica em objetivos e intervenções diferenciados.

\section{Fase Pós-Operatória Imediata}

Nesta, o objetivo primordial refere-se ao controle das condições gerais do indivíduo, evitando-se e detectando-se precocemente, eventuais complicações. No que tange ao estorna e efluente, o mesmo se aplica. A visualização constante do estorna, através de dispositivo adequado, e o. controle do efluente quanto 
ao volume e às características, são indispensáveis para a identificação de alterações dentre as quais, a isquemia parcial ou total do estorna, necrose e possivel afundamento da alça com conseqüente peritonite; hemorragias; obstruções, e distúrbios hidroeletrolíticos, no caso de ileostomias com alto débito (ERWIN-TOTH; DOUGHTY, 1992; FREITAS, 1994; CEZARETI et al, 1997; CREMA; MARTINS Jr, 1997).

\section{Fase Pós-Operatória Mediata}

Esta etapa é crítica no que se refere ao posicionamento do enfermeiro frente a um processo de avaliação adequado na fase pré-operatória. Tradicionalmente, segundo os autores, é nesta fase, a partir do $3^{\circ}$ dia pós-operatório, que se preconiza o ensino gradativo do autocuidado que envolve, inicialmente, a visualização e o toque precoces do estorna pelo paciente (ERWIN-TOTH; DOUGHTY, 1992; SANTOS, 1992; CEZARETI et al, 1997).

No entanto, deve-se reconhecer que o paciente pode encontrar-se experienciando uma fase de luto pela perda de parte corporal que implica, fundamentalmente, em perda de controle sobre as eliminações, consideradas como básicas do ponto de vista social e fisiológico; perda ou distorção da imagem corporal e da identidade prévias (KELLY; HENRY, 1993; SANTOS, 1996).

LINDEMANN (1944) em seu trabalho clássico sobre o luto e a privação, descreve algumas características similares ao processo de separação e perda, facilmente identificáveis na clientela ostomizada:

- distresses somático e sensorial, com comportamentos de exaustão, irrealidade e distância emocional;

- preocupação com a imagem do morto (ou, no caso do ostomizado, do segmento corporal perdido);

- culpa, raiva e hostilidade;

- perda de padrões e condutas.

Evidentemente, essas respostas são influenciadas pelo significado da perda, pelas reservas fisicas, por características de personalidade relacionadas à crenças, motivação, "locus" de controle interno ou externo e mecanismos de enfrentamento; além de fatores ambientais, todos estes levantados pelo enfermeiro no processo de avaliação pré-operatória.

PARKES (1972) apud KELLY (1985) descreve como fases do luto, a negação ou evitação_que evolui gradativamente para a admissão da perda; alarme, onde prevalece a ansiedade e o medo pelas conseqüências de perda e limitação das perspectivas futuras; busca, na qual embora predomine o pânico, já se vislumbram alternativas que, perpassadas pelos sentimentos de perda interior e mutilação, caminham para a resolução\} que se traduz na re-construção da identidade social.
Embasado nos conhecimentos destes conceitos, o enfermeiro deve identificar o processo de luto vivenciado pelo paciente, para então proceder ao ensino do autocuidado como forma de maximizar o controle e a segurança, favorecendo, já no hospital, o processo de re-construção da própria identidade. Em havendo maior permanência do paciente na etapa de negação, por características pessoais de "locus" de controle externo e mecanismos adaptativos predominantemente de evitação, deve-se compartilhar este cuidado com a familia através do elemento significativo já identificado previamente e que atuará como cuidador até uma fase de seguimento tardio. Casos em que esta etapa persiste indefinidamente, merecem encaminhamento para profissionais especializados.

A alta do ostomizado, como processo que se inicia no momento em que o indivíduo é internado, deve, portanto, ser manejada gradativamente, à medida que os problemas são identificados, as intervenções são planejadas e executadas e os resultados são avaliados. Não se deve esquecer que o momento da alta é crítico para o paciente e familiares, ao implicar no que denominamos de enfrentamento doméstico, que envolve significados como: "distância dos recursos humanos e materiais do hospital" e "ter que resolver os problemas com autonomia e independência". Daí a importância de se ter o paciente e família/ cuidador como centros da atenção e decisão, possibilitando-lhes recursos e alternativas que os tornem, minimamente, seguros e confiantes para a alta hospitalar.

Parte desses recursos consiste nos resultados do processo ensino-aprendizagem obtidos durante a hospitalização. Outra parcela é constituída pelos dispositivos cedidos em quantidade e qualidade suficientes e adequados, até a consulta ambulatorial ou domiciliar; pelo encaminhamento para Serviços de Assistência Especializada, atualmente existentes em diversos pontos do Estado de São Paulo e mesmo, em outros Estados da Federação, além dos recursos da comunidade como Associações de Ostomizados e grupos de auto-ajuda (SANTOS, 1992; CEZARETI et al, 1997).

\section{Fase Pós Operatória Tardia}

Esta constitui etapa fundamental ao garantir acompanhamento do cliente na continuidade de seu tratamento, no reforço e, muitas vezes, corno início do processo de ensino-aprendizagem do autocuidado; na adequação de dispositivos; na prevenção e tratamento de complicações; no treinamento de técnicas de irrigação e oclusão intestinal; no suporte para resolução de dúvidas na re-construção da imagem corporal, identidade e papéis; no seguimento da reinserção social paulatina, quer seja em nivel de atividades básicas de vida diária, ligadas ao autocuidado, quer seja nas 
atividades de trabalhos com ou sem realocação profissional, de lazer, associativas e sexuais, estas como um grande desafio tanto pelas dificuldades físicas conseqüentes às operações e tratamentos adequados como pelas crenças e tabus que circundam o assunto (SANTOS, 1992; CEZARETI et al, 1997).

\section{CONSIDERAÇÕES FINAIS}

A revisão sobre o processo de cuidar de ostomizados aqui apresentada buscou ampliar o que existe na literatura nacional, não só ao acrescentar alguns aspectos legais e éticos como norteadores da assistência, mas também ao questionar algumas "fórmulas/ rótulos" de atendimento já consagrados nas diversas fases operatórias, por incluir conceitos relacionados ao luto, ao "locus" de controle e mecanismos de enfrentamento, em direção à reconstrução da identidade pessoal e social.

Acredita-se que o suporte embasado em um trabalho interdisciplinar compartilhado e competente, em busca da otimização e potencialização dos recursos remanescentes do sujeito e do reconhecimento de novas possibilidades e caminhos, de ordem pessoal e institucional, em direção à efetividade de um processo reabilitatório realista, onde o paciente e a família/ elemento significativo, constituam o centro decisório, certamente possibilitarão o alcance do melhor nivel de qualidade de vida não só para esta clientela ostomizada como para todos aqueles cujo processo de cuidar esteja embasado nestes conceitos e princípios teórico-metodológicos.

\section{REFERENCIAS BIBLIOGRÁFICAS}

BRASIL, Leis etc. Lei n.7498 de 25 de junho de 1986. Dispõe sobre a regulamentação do exercício da Enfermagem e dá outras providências. IN: CONSELHO REGIONAL DE ENFERMAGEM. Documentos básicos de Enfermagem: enfermeiros, técnicos e auxiliares. São Paulo, s.d., p. $36-42$.

CASTLEDine, G. Are stoma nurses just bag handlers? Brit. J. Nurs., v.2, n.2, p.137, 1993.

CEZARETI, I. U. R. et al. A Enfermagem e o processo de cuidar de ostomizados. In: CREMA, E.; SILVA, R. Estomas: uma bordagem interdisciplinar. Uberaba, Editora Pinti, 1997. cap.9, p.125-44.

CONSELHO FEDERAL DE ENFERMAGEM. Resolução COFEN-160. Aprova o Código de Ética dos Profissionais de Enfermagem. IN: CONSELHO REGIONAL DE ENFERMAGEM. Documentos básicos de Enfermagem: enfermeiros, técnicos e auxiliares. São Paulo, s.d., p. 103-4.
CREMA, E.; MARTINS Jr, A. Complicações dos estomas intestinais. In: CREMA, E.; SILVA, R. Estornas: uma abordagem interdisciplinar. Uberaba, Editora Pinti, 1997. cap.7, p.89-106.

ERWIN-TOTH, P.; DOUGHTY, D. B. Principles and procedures of stomal management. In: HAMPTON, B. G.; BRYANT, R. A. Ostomies and continent diversions: nursing management. St Louis, Mosby Year Book, 1992. cap.1, p.1-28.

FREEDMAN, R. Meu corpo...meu espelho: aprendendo a conviver com seu corpo, a aceitar seu visual e a gostar cada vez mais de você. Rio de Janeiro, Rosa dos Tempos, 1994.

FREITAS, M. R. I. de. Subsídios para a compreensão da sexualidade do parceiro do sujeito portador de colostomia definitiva. Ribeirão Preto, 1994. $211 \mathrm{p}$. Dissertação (Mestrado) - Escola de Enfermagem de Ribeirão Preto, Universidade de São Paulo.

GOLDBERG, M. T. Promoting positive self-concept in patients with stomas: nursing interventions. Progressions, v.3, n.1, p.3-11, 1991.

KELLY, M. P. Loss and grief reactions as responses to surgery. J. Adv. Nurs., v.10, n.6, p.517- 25, 1985

KELLY, M. P. Patient's decision making in major surgery: the case of total colectomy. J. Adv. Nurs., v.19, n.6, p.1168- 7, 1994.

KELLY, M. P.; HENRY, P. Open discussion can lead to acceptance: the psychosocial effects of stoma surgery. Prof. Nurse, v.9, n.2, p.101-10, 1993.

LINDEMANN, E. Symptomatology and management of acute grief. Am. J. Psychi., v.101, n.2, p.141-8, 1944.

MILLER, J. F. Coping with chronic ilness: overcoming powerlessness. 2ed. Philadelphia, Davis, 1992. cap.1, p.3-17: Patient power resouces.

SANTOS, V. L. C. de G. Reabilitação do ostomizado: em busca do ser saudável. Texto Contexto Enf., v.1, n.2, p.180-90, 1992.

SANTOS, V. L. C. de G. A bolsa na mediação "estar ostomizado - estar - profissional": análise de uma estratégia pedagógica. São Paulo, 1996. Doutorado (Tese). Escola de Enfermagem, Universidade de São Paulo.

SMITH, D. B. Psychosocial adaptation. In: HAMPTON, B. G.; BRYANT, R. A. Ostomies and continent diversions: nursing management. St Louis, Mosby Year Book, 1992. cap.1, p.1-28.

STEGINGA, S. Stomalterapy: specialty nursing or special nursing? World Counc. Enterostom. Ther. J., v.13, n.4, p.8-9, 1993.

WORLD COUNCIL OF ENTEROSTOMAL THERAPISTSWCET/ An association of nurses. Members handbook. Australia, Ink Press International, 1998.

WRIGHT, K. A pragmatic view of the future. J. ET Nurs., v.20, n.3, p.89-90, 1993. 